
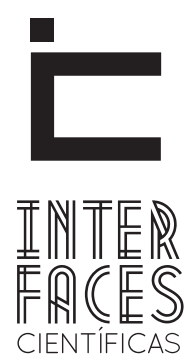

EXATAS E TECNOLÓGICAS

ISSN IMPRESSO - 2359-4934

ISSN ELETRÔNICO - 2359-4942

DOI-10.17564/2359-4942.2018v3n1p19-26

\title{
SENSOR DE TEMPERATURA VIA RASPBERRY PARA TRANSPORTE DE REFRIGERADOS
}

\author{
TEMPERATURE SENSOR OF RASPBERRY FOR REFRIGERATED TRANSPORT \\ SENSOR DE TEMPERATURA VIA RASPBERRY PARA EL TRANSPORTE DE REFRIGERADOS
}

Carlison Oliveira ${ }^{1}$

Rangers Colombo ${ }^{3}$

Washington Gimenes ${ }^{5}$

Thiago de Almeida Correia ${ }^{7}$
Marcelo Mattos ${ }^{2}$

Thiago Oliveira ${ }^{4}$

Bruno Silva Rodrigues ${ }^{6}$

\section{RESUMO}

O setor de transporte de refrigerados e congelados aumentam o lucro e fluxo para o comércio. As particularidades no transporte de carga refrigerada, geralmente tratam de produtos que podem sofrer qualquer tipo de deterioração, devido a climatização do ambiente não ser monitorada durante o transporte. A proposta do artigo é desenvolver um protótipo de monitoramento de temperatura e umidade para o transporte de refrigerados em geral. 0 protótipo apre- sentado utiliza componentes da nova revolução da computação, a Internet das Coisas, viabilizando para uma solução de baixo custo.

\section{PALAVRAS-CHAVE}

Transporte. Refrigerados. Internet das Coisas. Monitoramento. Temperatura. 


\section{ABSTRACT}

The refrigerated and frozen transportation sector increases profit and flow to trade. The particularities in the transport of refrigerated cargo generally deal with products that can suffer any type of deterioration due to the climatization of the environment not being monitored during transportation. The proposal of the article is to develop a prototype of monitoring of temperature and humidity for the transport of frozen in general.

\section{RESUMEN}

El sector de transporte de refrigerados y congelados aumenta el beneficio y el flujo para el comercio. Las particularidades en el transporte de carga refrigerada, generalmente tratan de productos que pueden sufrir cualquier tipo de deterioro, debido a que la climatización del ambiente no es monitoreada durante el transporte. La propuesta del artículo es desarrollar un prototipo de monitoreo de temperatura y humedad para el transporte de refrige-
The prototype presented uses components of the new computing revolution, the Internet of Things, enabling a low-cost solution.

\section{KEYWORDS}

Transport. Refrigerated. Internet of Things. Monitoring. Temperature. rados en general. El prototipo presentado utiliza componentes de la nueva revolución de la computación, la Internet de las cosas, viabilizando para una solución de bajo costo.

\section{PALABRAS CLAVE}

Transporte; refrigerada; congelados; Internet de las cosas; monitoreo; la temperatura; 


\section{INTRODUCÇÃO}

O setor de transporte de refrigerados e congelados aumenta o lucro e fluxo para o comércio, isso devido à grande variedade de produtos existente e levados ao consumidor. De acordo com a Associação Brasileira das Indústrias de Alimentação ( $A B \mid A$ ) o mercado de refrigerados e congelados prontos para consumo atingiu $\mathrm{R} \$$ 7,4 bilhões de reais em 2011, sendo que o resultado foi de R\$ 6,3 bilhões de reais em 2010 (NEW TRADE, 2017).

Distribuir alimentos perecíveis torna a logística fundamental no anseio dos consumidores de exigir cada vez mais qualidade nos alimentos, atributo essencial para o comércio. As particularidades no transporte de carga refrigerada, geralmente tratam de produtos que podem sofrer qualquer tipo de deterioração, seja biológica, física ou química e que prejudicam suas qualidades para industrialização, comercialização e consumo. 0 desafio desse tipo de transporte assegura que a mercadoria será transportada no ambiente adequado e contínuo ao longo do seu percurso, garantindo sua qualidade durante todos os processos envolvidos como armazenamento, distribuição, transporte e consumo (REZENDE, 2011).

Desde 29 de junho de 2002 a norma NBR 14701, estabelece critérios de temperatura para 0 transporte de produtos alimentícios refrigerados. Seu objetivo é manter uma temperatura adequada ao longo de toda a cadeia de abastecimento, desde os armazéns frigoríficos até a entrega do produto no varejo. A norma abrange embalagem, movimentação, preparação de docas, uso de registradores de temperatura nos estoques e nos transportes, entre outros (ABNT, on-line).

A fim de aprimorar a tecnologia de verificação de temperatura local no transporte de alimentos congelados e resfriados, este artigo apresenta um projeto conceitual de melhoria na solução de monitoramento da temperatura do ambiente interno do compartimento de carga dos caminhões refrigerados. A solução que conecta o produtor à indústria, possibilitando acompanhar a temperatura de seus produtos durante o transporte, obedecendo às normas estabelecidas pelo mercado para que o produto se mantenha dentro dos padrões de qualidade aceitável.
As próximas seções deste artigo estarão organizadas da seguinte maneira: a seção 2 apresentará a fundamentação teórica. A seção 3 descreverá os métodos e funcionamento utilizados para o desenvolvimento da solução de monitoramento. A seção 4 apresentará os resultados dos experimentos obtidos. A seção 5 as considerações finais.

\section{FUNDAMENTAÇÃO TEÓRICA}

Nos próximos anos "bilhões de coisas" distribuídas e inteligentes estarão conectadas para disponibilizar novos tipos de aplicativos, serviços e formas de interação (ALKHABBAS, 2017). A Internet of Things (IOT) é um novo conceito de comunicação destinado a conectar essas "coisas" (sensores, microcontroladores e transceptores) para comunicação digital que possibilitará a interação uns com os outros, obtendo dados e funcionalidades aos usuários por meio da Internet (ZANELLA, 2014).

O loT está em ascensão, tanto no seu desenvolvimento quanto na sua aplicação cada vez mais comum no dia a dia. Tomando por base a perspectiva da revista Harvard Business Review, a utilização de tecnologia baseada em loT no Brasil deve alcançar um crescimento de US\$ 39 bilhões no PIB até o ano de 2030 (PURDY, 2015).

Alguns componentes (FIGURA 1) que formam uma IoT, são chamados nós sensores e esses constituídos por um conjunto de hardware, são eles: memória, bateria, processador, transmissor e sensor (MACEDO, 2005).

Figura 1 - Principais componentes de um nó sensor

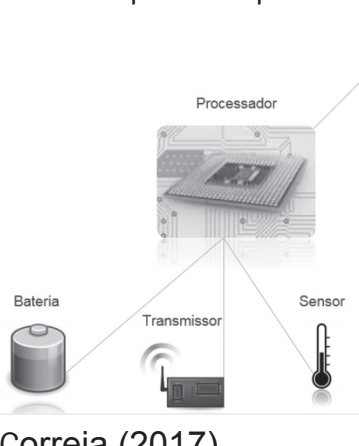

Fonte: Correia (2017). 
A memória é a área responsável pelo armazenamento dos dados de forma temporária. A bateria uma fonte de energia para alimentar os componentes elétri$\cos$ (TILAK, 2002). 0 processador responsável pelo processamento de dados e controle das funcionalidades dos componentes do NS. O transmissor responsável pelo envio e recepção dos dados (BRAGA, 2010). E por último o sensor que normalmente são componentes pequenos sensíveis a detecção de algumas formas de energia, tais como luminosa, térmica e cinética, temperatura, corrente, entre outras (THOMAZINI, 2011).

\section{METODOLOGIA E FUNCIONAMENTO}

A proposta do artigo é desenvolver um protótipo de monitoramento de temperatura e umidade interna de um baú refrigerado. Os sensores de temperatura e umidade devem ser instalados internamente no baú e conectá-los a central de processamento local (CPL), instalada na cabine do caminhão, para permitir que o motorista visualize as informações obtidas pelos sensores e disponibilizá-las na Internet para um acompanhamento em tempo real.

O sensor instalado no baú realizará a leitura da temperatura e umidade simultaneamente e esse conectado a CPL para que esses dados possam ser processados e disponibilizados em um dashboard. A CPL possui os seguintes componentes de hardware:

1) Raspberry: um microcomputador completo com entrada para cartões de memória, USB, HDMI, memória RAM e módulo de transmissão Wi-Fi e Bluetooth;

2) Sensores de temperatura e umidade: o DHT-22 é uma versão que trabalha na faixa de temperatura estabelecida pelas normas e regulamentos da Agência Nacional de Vigilância Sanitária (ANVISA) e Associação Brasileira de Normas Técnicas (ABNT);

3) Tela de liquid crystal display (LCD): tela de cristal líquido modelo touch screen conectada ao Raspberry para visualizar as leituras de temperatura e umidade.

O fluxo da informação na solução pode ser visualizado na Figura 2 e descrito em detalhes na sequência.
Figura 2 - Fluxo da informação da solução proposta

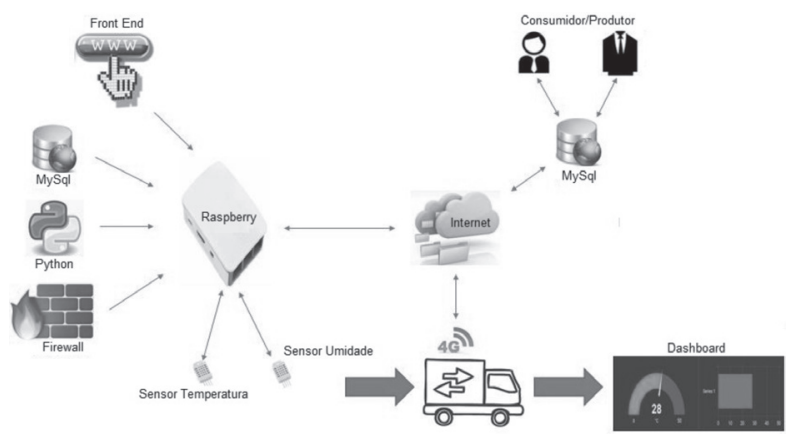

Fonte: Elaborado pelos autores

O caminhão de transporte de alimentos congelados e resfriados, que tem a solução embarcada.

O Raspberry é a CPL, pois concentra todos os softwares (MySql, Python, Firewall) utilizados, além de ser o responsável pelo processamento da informação e disponibilização desses localmente e via Internet.

A nuvem representa a Internet, que disponibiliza uma página web com as informações dos sensores, ou também poderá receber essas informações via e-mail.

O dashboard possibilita a visualização e acompanhamento da temperatura e umidade ao longo de todo transporte. Os dados são disponibilizados localmente para o motorista e via internet, também, podendo visualizar por meio de um dashboard.

O funcionamento da solução consiste na leitura de temperatura e umidade que serão enviados ao banco de dados do CPL. Essa rotina de leitura foi programada para ser executada a cada um minuto e para uma melhor visualização e interpretação desses dados foi utilizado um dashboard, além de ser possível o envio desses dados por meio da Internet. Os dados disponibilizados na Internet podem ser registrados em outro banco de dados externo, para diversas finalidades, como por exemplo a geração de um relatório.

A solução de banco de dados é um software aberto e livre, o MySQL. Uma vantagem de utilizar um banco de dados possibilita uma resposta mais rápida no pedido das informações, que é crucial nos dias de hoje, os clientes possuem uma forma de registrar e captar os da- 
dos assincronamente, uma quantidade de informações integradas, verificada com maior agilidade, mostrando os resultados expressivos com base em seus dados.

0 acesso ao banco de dados é feito por meio do interpretador PHP, que disponibilizará essas informações em uma página web HTML. O PHP é uma linguagem de script open source de uso geral, muito utilizada e, especialmente, adequada para o desenvolvimento web e que pode ser embutida dentro do HTML.

Para a exibição da dashboard na tela, foi utilizado um programa denominado Node-Red, uma ferramenta de programação para conectar dispositivos de hardware, APIs e serviços on-line de maneiras novas e interessantes.

No CPL foi instalado o sistema operacional $\mathrm{L} i$ nux Debian 8 com a biblioteca Adafruit_Python_ DHT para realizar a leitura dos dados dos sensores de temperatura e umidade. Uma rotina programada e agendada pelo crontab foi necessária, para que os dados possam ser acessados no banco de dados e enviados a Internet por meio de um modem $4 \mathrm{G}$, por exemplo, mas que pode ser substituído por outra tecnologia Global System for Mobile Communications (GSM). A visualização dos dados na Internet, só é possível por meio de uma conta de e-mail. 0 projeto utilizou um cliente de e-mail do Linux para encaminhar os dados em uma conta do Gmail.

\section{RESULTADOS}

A principal função da CPL baseia-se em coletar dados por meio do sensor de temperatura e apresentar seus resultados no dashboard e Internet para fácil visualização em uma página web de qualquer lugar, além de enviar por e-mail no caso de alteração de temperatura especificada.

Depois de implementado o equipamento no caminhão pela fornecedora basta ligar o caminhão e aguardar o sistema iniciar e será apresentado no dashboard as informações sobre temperatura e umidade (FIGURA 3).

Figura 3 - Dashboard de temperatura

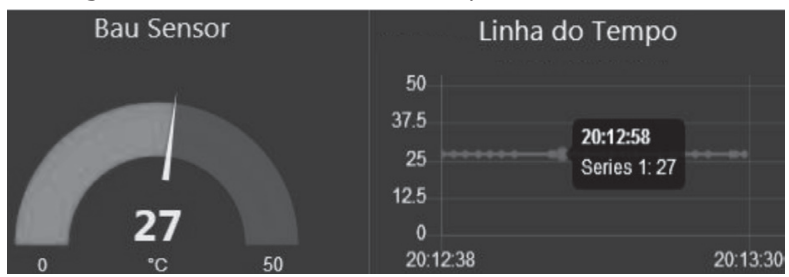

Fonte: Elaborado pelos autores

As informações de temperatura e umidade também podem ser disponibilizadas via e-mail, conforme Figura 4.

Figura 4 - Informações de temperatura por e-mail

\section{Grupo lotech de Monitoramento da Temperatura de Transportes Logístico}

\begin{tabular}{|lll|}
\hline Data & Sensor & \multicolumn{2}{l}{ Temperatura Humidade } \\
2017-09-15 20:00:01 & Raspberry temperatura 27.1 & 46.2 \\
2017-09-15 20:10:02 & Raspberry temperatura 27.1 & 46.5 \\
2017-09-15 20:20:01 & Raspberry temperatura 26.8 & 46.5 \\
2017-09-15 20:30:02 Raspberry temperatura 26.5 & 47.6 \\
2017-09-15 20:40:01 Raspberry temperatura 26 & 50.5 \\
2017-09-15 20:50:02 Raspberry temperatura 25.9 & 50.9 \\
\hline
\end{tabular}

Todos os direitos reservados ao Grupo lotech de monitoramento 2017

Fonte: Elaborado pelos autores 
Foram realizados testes de funcionamento do equipamento, estando em um ambiente fechado de aproximadamente $29^{\circ} \mathrm{C}$, utilizando um copo de isopor com gelo para manter a temperatura no ambiente interno do copo. Depois alteramos as configurações da temperatura para no mínimo $10^{\circ} \mathrm{C}$ e máxima de $21^{\circ} \mathrm{C}$, após atualização dos parâmetros inseridos no equipamento e colocar o sensor no copo de isopor com gelo, a diminuição da temperatura foi constante com um tempo aproximado de 2 minutos, até a marca de $8.5^{\circ} \mathrm{C}$, já acionando o alerta de fora da conformidade pois estava abaixo do mínimo configurado.

Em seguida verificamos os envios dos e-mails as partes interessadas, ou seja, as pessoas que irão fazer o acompanhamento da qualidade da mercadoria, mostrando que o sistema funciona conforme planejado pela equipe, lembrando que o recomendo pela norma NBR 14701 a câmara fria ou baú deve estar estabilizada obrigatoriamente pelo menos 15 minutos antes da entrada do produto.

Também foram feitos os testes de temperatura máxima, apenas retirando o sensor do copo com gelo e mantendo os parâmetros da temperatura de mínima de $10^{\circ} \mathrm{C}$ e máxima de $21^{\circ} \mathrm{C}$ onde obtivemos o aumento da temperatura, chegando a $23.1^{\circ} \mathrm{C}$ em aproximadamente 2 minutos, desde a retirada até a identificação do sensor que imediatamente avisou por meio de e-mails a condição de fora de conformidade dos padrões, mas de final chegou a $29.3^{\circ} \mathrm{C}$ após 6 minutos que era a condição atual do local onde eram feitos os testes.

Assim, foi possível verificar pelo site se os dados coletados foram armazenados no banco de dados Mysql e se era possível acessar por meio do celular a página da solução, que nesse caso ambos se mostraram funcionando como esperado.

\section{CONCLUSÃO}

O objetivo deste projeto conceito é atender o setor de transporte logístico de resfriados em geral, que é um dos mercados com forte impacto econômico no país, além de crescimento constante devido ao consumo interno e exportações, assim movimenta uma grande fatia do PIB do Brasil. Com esse mercado em expansão, cresce a necessidade de se fazer o monitoramento de temperatura simultâneo de baús de caminhões dessas transportadoras, a fim de melhorar a qualidade do serviço e ajudar no crescimento do mercado.

A solução de monitoramento proposta neste artigo, oferece ao produtor, a indústria e as transportadoras 0 monitoramento da temperatura, mantendo os padrões de qualidade estabelecidos pelos órgãos competentes.

Nesse primeiro contato foi possível reunir bases para conhecimento e aplicação ao trabalho e desenvolvimento do projeto, como, por exemplo, as tecnologias que serão utilizadas, normas que regulamentam esse tipo de serviço, regras e pesquisas feitas sobre o mercado e sua perspectiva de grande crescimento para os próximos anos.

Com esses dados reunidos e pesquisas feitas, com a ajuda da parte de desenvolvimento dentro do processo de criação do projeto, foi possível criar um Minimum Viable Product (MVP) ou Projeto Mínimo Viável que corresponde a construção de peças vitais para o equipamento funcionar.

\section{REFERÊNCIAS}

ABNT. Catálogo. Disponível em: <http://www. abntcatalogo.com.br>. Acesso em: 15 abr. 2017.

ALKHABBAS, D.P.F.; SPALAZZESE, R. Architecting emergent configurations in the internet of things. IEEE Int. Conf. Softw. Archit., 2017. p.221-224.

\section{BRAGA, T.C. Monitorização ambiental em espaços} florestais com rede de sensores sem fios. 2010. Tese (Doutorado) - Universidade da Madeira, 2010.

\section{CORREIA, T.A. RSSF para detecção de incêndios}

florestais em tempo real. 2017. Dissertação

(Mestrado) - PUC, Campinas-SP, 2017.

MACEDO, D.F. et al. Um protocolo de roteamento configurável baseado em regras para redes de 
sensores sem fio. $23^{\circ}$ Simpósio Brasileiro de Redes

de Computadores. 2005, p.89-102.

\section{NEW TRADE. Cresce a preferência do consumidor por produtos congelados. 2017. Disponível}

em: <http://www.asbra.com.br/noticia/crescea-preferencia-do-consumidor-por-produtoscongelados>. Acesso em: 25 abr. 2017.

PURDY, M; DAVARZANI, Ladan; OVANESSOFF, Armen. Como a Internet das Coisas pode levar à próxima onda de crescimento no Brasil. 2015. Disponível em: <http://hbrbr.uol.com.br/como-a-internet-dascoisas-pode-levar-a-proxima-onda-de-crescimentono-brasil/>. Acesso em: 25 abr. 2017.
REZENDE, Antonio Carlos da Silva. Notícias, movimentação. Revista Logística, 2011. Disponível em: <https://www.imam.com.br/logistica/noticias/ movimentacao/107-logistica-de-distribuicao-dealimentos-pereciveis>. Acesso em: 20 abr. 2017.

THOMAZINI, D.; ALBUQUERQUE, P.U.B. Sensores industriais fundamentos e aplicações, 8th. Érica, 2011.

TILAK, S., ABU-GHAZALEH, N.B.; HEINZELMAN, W. A taxonomy of wireless micro-sensor network models. ACM SIGMOBILE Mob. Comput. Commun. Rev., v.6, n.2, p.28-36, 2002.

ZANELLA, A. Internet of things for smart cities. IEEE Internet of Things journal, v.1, n.1, p.22-32, 2014. 
